\title{
Profiling Skype Video Calls: Rate Control and Video Quality
}

\author{
Xinggong Zhang*, Yang $\mathrm{Xu}^{\dagger}$, Hao $\mathrm{Hu}^{\dagger}$, Yong $\mathrm{Liu}^{\dagger}$, Zongming Guo* and Yao Wang ${ }^{\dagger}$ \\ ${ }^{*}$ Institute of Computer Science\&Technology, Peking University, Beijing 100871 \\ ${ }^{\dagger}$ Electrical \& Computer Engineering, Polytechnic Institute of NYU, Brooklyn, NY 1120
}

\begin{abstract}
Video telephony has recently gained its momentum and is widely adopted by end-consumers. But there have been very few studies on the network impacts of video calls and the user Quality-of-Experience (QoE) under different network conditions. In this paper, we study the rate control and video quality of Skype video calls. We first measure the behaviors of Skype video calls on a controlled network testbed. By varying packet loss rate, propagation delay and available network bandwidth, we observe how Skype adjusts its sending rate, FEC redundancy, video rate and frame rate. We find that Skype is robust against mild packet losses and propagation delays, and can efficiently utilize the available network bandwidth. We also find that Skype employs an overly aggressive FEC protection strategy. Based on the measurement results, we develop rate control model, FEC model, and video quality model for Skype. Extrapolating from the models, we conduct numerical analysis to study the network impacts of Skype. We demonstrate that user back-offs upon quality degradation serve as an effective user-level rate control scheme. We also show that Skype video calls are indeed $T C P$ friendly and respond to congestion quickly when the network is overloaded.
\end{abstract}

\section{INTRODUCTION}

The Internet has fundamentally changed the way people communicate. While emails, text-messages, and Voice-overIP (VoIP) calls will remain to be important communication means, we are at the threshold to embrace the next big change: Video Telephony. Most online chatting software, such as MSN messenger, GTalk and Facetime support video chat. Skype [1], arguably the most popular audio/video communication platform for end-consumers, provides two-way video calls and multi-party video conferencing to its 500 million users on PCs or mobile devices. Video calls are much more bandwidthdemanding than voice-over-IP (VoIP) calls. While the data rate of a typical Skype VoIP call is around $30 k b p s$, as will be shown shortly, a good quality Skype video call can easily consume bandwidth up to $950 \mathrm{kbps}$, representing an increase by a factor of more than thirty. The sheer traffic volumes generated by video calls make it imperative for network providers and network researchers to understand the impact of this new "killer application" on the performance and stability of the existing network protocols and infrastructures. Meanwhile, due to the real-time interaction between users, the quality of a video call is more sensitive to packet losses and delays than one-way video streaming. It is therefore of great interest for end users and application developers to assess the quality of video calls under different network conditions. However, up to now, there have been very few study on the popular video telephony applications.
In this paper, we present our recent effort on profiling Skype video calls' rate control and video quality through empirical measurement and analytical modeling. We attempt to answer two key questions:

Q1: How does a Skype video call adapt its sending rate, video rate and quality under different network conditions?

Q2: Are Skype video calls friendly to TCP flows when they compete for network resources?

It is challenging to come up with comprehensive answers for those questions. First of all, Skype is a proprietary software. There is very limited public information about its video encoding and transmission algorithms. The common practice is to treat it as a black-box and observe its behaviors under different conditions. Secondly, real network conditions are highly diverse, often time-varying and traffic-varying. It is impossible to come up with a set of scenarios that are representative for all networks. Measuring Skype in a fast changing network environment often gives misleading conclusions.

To address those challenges, we answer $Q 1$ using an extensive measurement study of Skype in a controlled environment. We set up a network testbed with configurable packet loss, propagation delay, and available link bandwidth. We systematically generate different network settings. In each setting, we measure Skype's sending rate, throughput, round-trip time (RTT), video bit rate, and frame rate after it reaches the steady state. Based on the measurement data, we propose rate control model, FEC model, and video quality model for Skype video calls. Those models enable us answer $Q 2$ quantitatively. Specifically, we study the effectiveness of user back-offs upon quality degradation as a user-level rate control scheme. We study the TCP-friendliness of Skype video calls by comparing its responsiveness to packet losses and delays with TCP. We also conduct a numerical case study on a $4 \mathrm{G}$ type of wireless network where multiple Skype flows and TCP flows compete for bandwidth on uplink.

The contributions of our study are two-fold:

1) We are the first to measure the stationary behaviors of Skype video calls under different network conditions. We have the following findings: a) Skype's sending rate is insensitive to packet losses when the packet loss rate is below 10\%; Skype significantly reduces its sending rate when loss rate goes above 10\%; b) Skype keeps close track of the available network bandwidth, and can maintain bandwidth utilization around $80 \%$ under a wide range of network conditions; c) Skype's sending rate is 
insensitive to propagation delays; d) Skype utilizes an overly aggressive FEC scheme. Its FEC redundancy ratio is about 4.5 times the actual packet loss rate.

2) We develop models for Skype's rate control, FEC redundancy, and video quality. Using the models, we demonstrate that user back-offs react fast to the onset of congestion, and serve as an efficient user-level rate control mechanism. We are the first to include user behaviors in studying rate control of video calls. We further show that, with the built-in rate control scheme and quality-driven user back-offs, Skype video calls are indeed $T C P$-friendly, and are very responsive to congestion when the network is overloaded.

The rest of the paper is organized as follows. Section II describes the related work. We present our measurement methodology and testbed configurations in Section III. In Section IV, we present measurement results on the stationary behaviors of Skype. In Section V, we propose a rate control model, a FEC model and a video quality model for Skype. In Section VI, we study Skype's TCP-friendliness by taking into account user back-offs. Finally, we conclude the paper with summary and future work in Section VII.

\section{RELATED WORK}

Measurement work on Skype can be classified into two categories: characterizing network protocols and exploring VoIP details. Baset et al [2] first analyzed Skype's P2P topology, call establishment protocols, and NAT traversal mechanism. By analyzing Skype call traffic, they reverse engineered the communication mechanisms of Skype. Since then, a lot of papers have been published on Skype's overlay architecture, $\mathrm{P} 2 \mathrm{P}$ protocol, and VoIP traffic [3], [4]. In the second category, some studies [5], [6], [7] focused on the quality of Skype's voice-over-IP (VoIP) calls. Huang et al. investigated Skype's FEC mechanism and its efficiency for voice streaming [6], [7]. In [5], the authors proposed a USER Satisfaction Index model to quantify VoIP user satisfaction. Cicco et al. [8] proposed a congestion control model for Skype VoIP traffic. All of these studies only focused on the voice service of Skype, did not consider its video service.

In a closely related paper, Cicco et al. [9] measured the responsiveness of Skype video calls to bandwidth variations. They conclude that Skype's response time to bandwidth increase is long. However, they only presented some empirical data, and did not systematically measure and model the stationary behaviors of Skype. We conducted extensive measurement of Skype under different network settings of packet losses, packet delays and available network bandwidth. Based on the measurements, we propose the models for Skype video calls' rate control, FEC redundancy, and video quality. There have been some other related studies on investigating the impact of user behaviors on network stability [10], [11]. In [11], Tay et al. studied how TCP user aborts enable a network sustain a higher demand without causing congestion collapse. Bu et al. proposed in [10] that the user back-offs in VoIP will help maintain the network stability. They assumed that VoIP flows don't adapt their sending rates to congestion. Instead, they showed that user back-off is an efficient userlevel congestion control mechanism for VoIP. We study rate control of Skype video calls. In our experiments, we observe that Skype video call has a built-in rate control mechanism, which adapts its video rates to network conditions in a wide range. On top of that, we show that user back-offs can further enhance Skype video calls' responsiveness to network congestion.

\section{Measurement Test-Bed Setup}

To study Skype under various network conditions, we set up a controlled testbed in Fig. 1 on which Skype is observed as a black-box. Two clients with Skype Ver. 5.2 are connected by a NAT router. Each host has a private IP address and connects to the Internet through the router. To emulate a wired or wireless network, all packets pass through a software-based network emulator, NEWT[12]. It emulates a variety of network attributes, such as propagation delay, random packet loss, and available bandwidth. We also inject UDP background traffic into the testbed using iPerf tools. To emulate a video call,

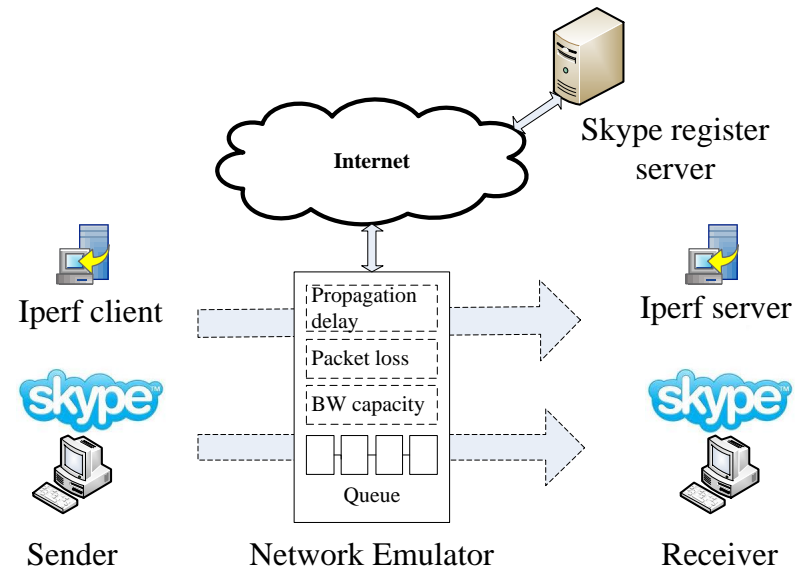

Fig. 1: The network setup for Skype measurement

we choose a standard TV news video sequence "Akiyo" from JVT (Joint Video Team) test sequence pool. The sequence has mostly head and shoulder movements. It is very similar to a video-call scenario. We inject the video sequence into Skype using a virtual video camera tool [13]. This ensures the transmitted video content are consistent and repeatable.

The measurement data are collected by two methods: TCPdump for packet level information, and Skype reports for video level information. Since Skype employs proprietary protocols and encrypts all control messages, it is hard to reverse-engineer its protocols and measure video information externally. Fortunately, Skype reports some technical information through its user interface, such as video rates, frame rates, RTT, et al. We use a screen text capture tool [14] to capture these information periodically from Skype window. The sampling interval is 1 second. Unlike previous work [9], we are more interested in Skype's stationary behaviors. By giving enough time for Skype to adapt to a given network scenario, we are able to analyze its built-in rate control mechanisms. In each 
TABLE I: Measured Video Parameters of Skype Codec

\begin{tabular}{|l|c|}
\hline Video Resolution & $640 * 480,320 * 240,160 * 120$ \\
\hline Frame Rate per Second & 5fps - 30fps \\
\hline Video Bit Rate & 30kbs - 950kbs \\
\hline
\end{tabular}

scenario, after Skype enters steady state, we capture data from ten minutes of a video call. To cope with random effects, we report the mean and $95 \%$ confidence interval for each data point of interest.

Skype uses two transport layer protocols: TCP protocol for control messages and UDP protocol for video transmission. The TCP connection acts as a feedback channel through which the receiver periodically reports current network conditions to the sender. Then the sender adapts its UDP sending rate to network conditions. According to our observations, a typical signaling protocol for a video call is as follows: 1) Skype clients connect to a register server on the Internet to log into the system. 2) To start a video call, a client first sends a message to the register servers and looks up the IP address of the callee. 3) The caller attempts to establish a call session with the callee either directly or through a relay. In our experiments, even though the two hosts are behind a NAT router and are assigned with private IP addresses, the caller is always able to find the correct private address of the callee and connect to the private address directly without going through a relay. The monitored video call only traverses the network emulator and NAT router before it reaches the callee. We have full control on the network condition along the path.

Skype employs a video codec provided by On2 [15]. It is a non-scalable motion-estimation based video codec. In our experiments, the video encoder version of VP6, VP7, and VP8 have ever been detected. It demonstrates Skype is embedded with multiple versions of video codec, and is compatible with earlier versions. This codec supports real-time video coding to generate variable video bit rates. It adapts its rate to network conditions by adjusting video quantization step, video resolution, and the number of frames per seconds (FPS). The observed video codec parameters in the measurements are listed in Table I.

\section{Measurement Results}

In this section, we report measured Skype performances under various network conditions. By isolating various network impairments, we investigate how Skype adapts its sending rate and video rate to packet loss, packet delay and available bandwidth.

\section{A. Impact of Packet Loss}

We first introduce random packet losses using the network emulator. The sending rate, RTT, and video rate of Skype are measured when Packet Loss Rate (PLR) varies from $0 \%$ to $12 \%$. The two-way propagation delay is fixed at $50 \mathrm{~ms}$. To examine whether Skype's responses to packet loss is consistent, we also carry out the above experiments under three different available bandwidth settings: $250 \mathrm{kbps}, 750 \mathrm{kbps}$, and $1000 \mathrm{kbps}$. The measurement results are illustrated in Figure 2. Figure 2(a) plots the mean and $95 \%$ confidence interval of

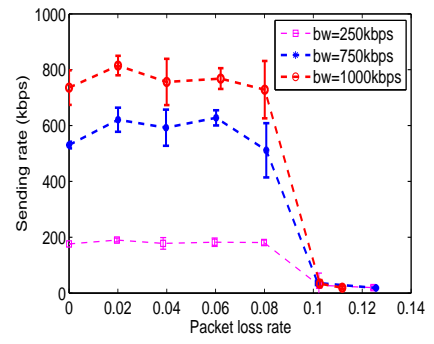

(a) Sending Rate

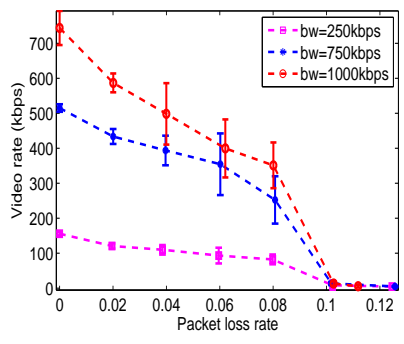

(b) Video Rate
Fig. 2: Impact of packet losses under different link bandwidth.

Skype sending rate. The mean and confidence interval of video rate are plotted in Figure 2(b). First, when PLR is less than $10 \%$, Skpye's sending rate almost remains constant while its video rate drops almost linearly with PLR. The gap between the sending rate and the video rate increases with PLR. We conjecture that this gap is due to the Forward Error Correction (FEC) coding. It has been reported in [7] that Skype implements FEC to combat packet losses for voice calls. It is reasonable to assume it protects video calls against packet losses in a similar way. As PLR increases, Skype reduces its video rate and allocates more bandwidth to FEC packets. We will further verify this assumption in Section V.

When PLR is larger than $10 \%$, both sending rate and video rate drop dramatically under the three bandwidth settings. Even though there is still abundant bandwidth available on the path, Skype still drops its sending rate to the lowest rate. It indicates that these drops are not due to network congestion. Thus, we infer that Skype operates in two states: Normalstate (NORM) and Conservative-state (CONS). Whenever it detects that the network condition becomes very bad, it would switch to the CONS state. In this state, Skype only sends out necessary data at a low constant rate. From Figure 2, we know $P L R \geq 10 \%$ is one trigger for Skype to switch to CONS state. We observe that in NORM state, the sending rate oscillates around $500 \mathrm{kbps}$ under link bandwidth of $750 \mathrm{kbps}$. The trend is the same for the other two link bandwidth settings. This fact indicates that in NORM state, the sending rate of Skype is ignorant to packet losses. This is totally different from the widely used TCP congestion control scheme, that reduces the sending rate by half upon each perceived packet loss. This immediately raises the concern that whether Skype is TCPfriendly. We will come back to this issue in Section VI.

From this study, we observe that when the packet loss rate is below 10\%, Skype works in normal (NORM) state, in which its sending rate is loss-ignorant, and its video rate decreases proportionally to loss rate; Skype switches to conservative (CONS) state whenever the packet loss rate goes over $10 \%$. Both its sending rate and video rate will be significantly reduced to a low value.

\section{B. Impact of Available Bandwidth}

To investigate how Skype responds to bandwidth available in the network, we vary the bandwidth capacity on the network emulator from 50kbps to $1000 \mathrm{kbps}$ while fixing the PLR and propagation delay. In order to cover both NORM state and 


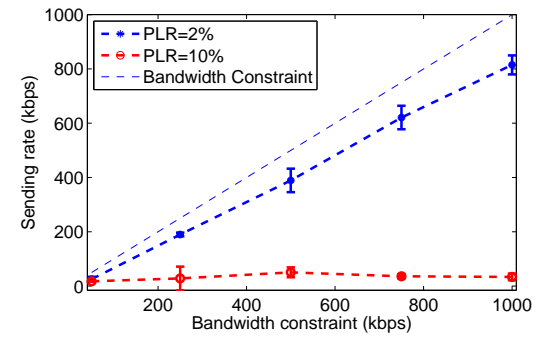

(a) Sending Rate

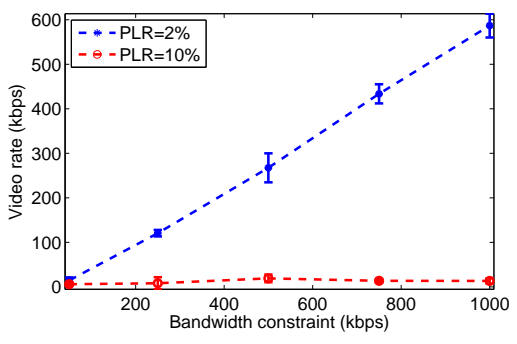

(b) Video Rate

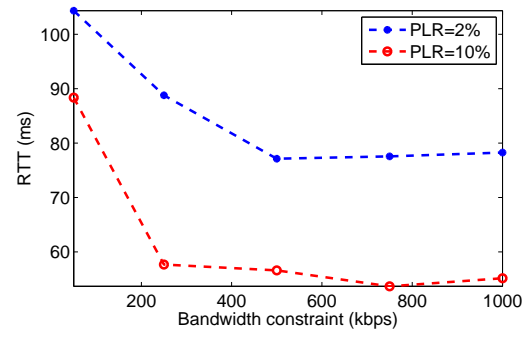

(c) Round-trip time

Fig. 3: Impact of available network bandwidth for PLR $=2 \%$ and $10 \%$. The two-way propagation delay is fixed at $50 \mathrm{~ms}$.

CONS state, two PLRs are considered, $2 \%$ and $10 \%$. In both cases, the two-way propagation delay is set to $50 \mathrm{~ms}$. The measured results are shown in Figure 3. We again observe distinct behaviors of Skype in NORM state and CONS state. In Figure 3(a), when $P L R=2 \%$, Skype is in NORM state and increases its sending rate proportionally as the available bandwidth increases. On the other hand, when $P L R=10 \%$, Skype is in CONS state and its sending rate almost remains unchanged even when the available bandwidth increases. This verifies our conjecture in the last section that Skype has two states. When it detects PLR is larger than $10 \%$, it will switch to the CONS state and sends data at the lowest rates. Similar trend can be detected in video rate as illustrated in Figure 3(b): video rate in NORM state changes linearly with available bandwidth, and remains unchanged in CONS state. It is also noticed that the video rate increases proportionally with the sending rate. There exists a linear function between sending rate and video rate. We will come back to study this in Section V-B.

The changes of RTT with the available bandwidth are shown in Figure 3(c). We observe that RTT decreases in general as available bandwidth increases. The slight increasing trend towards the end of the curves is due to the variations of RTT and the curves only report the mean. With the fixed two-way propagation delay, the RTT reduction is due to the queueing delay decrease resulted from the available bandwidth increase. We will quantitatively study the relation between RTT, sending rate and available bandwidth in Section V-A.

From these results, we find that Skype can closely keep track of the available network bandwidth and adjust its sending rate and video rate to efficiently utilize available bandwidth without causing excessive congestion. While the exact algorithm employed by Skype to track available bandwidth is unknown, due to its loss-ignorance in NORM state as studied in Section IV-A, we conjecture that the algorithm is most likely driven by packet delays, as commonly done in the available network bandwidth measurement [16], [17].

\section{Impact of Propagation Delay}

Network delay perceived by a packet consists of two components: congestion delay and propagation delay. While congestion delay is highly variable, propagation delay is static and is determined by the route taken by the packet. In this section, we measure Skype's performance by varying the two-way propagation delay in the network emulator. The
TABLE II: Mean value of Skype performances under various propagation delay.

\begin{tabular}{|l|c|c|l|}
\hline Propagation delay & Sending rate & Video rate & RTT \\
\hline 50 & 354.4 & 343.6 & 64.9 \\
\hline 200 & 358.7 & 342.3 & 220.5 \\
\hline 400 & 352.7 & 335.9 & 416.1 \\
\hline 1000 & 355.8 & 338.9 & 1017.7 \\
\hline 2000 & 368.8 & 350.9 & 2019 \\
\hline
\end{tabular}

bandwidth capacity is set to 500kbps and the PLR is 0 . We vary the propagation delay from $50 \mathrm{~ms}$ to $2000 \mathrm{~ms}$.

The measurement results are listed in Table II. We observe in this scenario Skype keeps sending rate stable around $360 k b p s$ for all propagation delay settings. We infer that typically Skype does not adapt its sending rate when the propagation delay changes. The RTT observed by Skype increases linearly with propagation delay. The small differences between the two is due to the queueing delay occurred in the network.

From the results, we conclude that Skype rate control is insensitive to propagation delay. ${ }^{1}$ Combined with the findings in Section IV-A and IV-B, we conjecture that Skype's rate control algorithms are driven by the congestion delay, instead of the propagation delay.

\section{Modeling Skype Behaviors}

In this section, we present more extensive measurement results and propose analytical models for Skype's sending rate, video rate, and video quality. Those models will allow us to extrapolate the measurement results to quantitatively answer important questions regarding the effectiveness of user backoffs as a rate control mechanism and the competition between Skype video calls and TCP flows in Section VI.

\section{A. Sending Rate}

In Section IV, we showed that Skype increases its sending rate linearly with the available bandwidth. In addition, Skype's rate control is insensitive to packet losses in both NORM and CONS states. We propose to model Skype's sending rate $R_{S}$ as a piecewise linear function as follows.

$$
R_{S}\left(C_{w}, p\right)= \begin{cases}\gamma C_{w}+\mu & p<10 \% \\ \delta & p \geq 10 \%\end{cases}
$$

\footnotetext{
${ }^{1}$ Due to the realtime interaction requirement, when the propagation delay is excessively long, e.g. over 2 seconds, users would have dropped the video call. That will effectively reduce Skype video call traffic.
} 


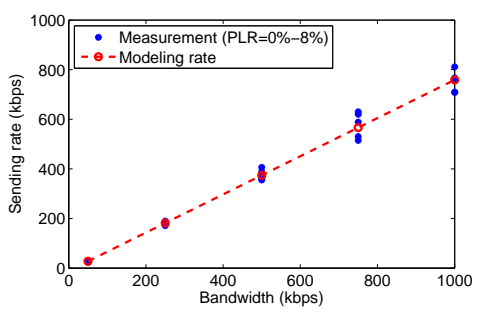

(a) Skype in NORM State

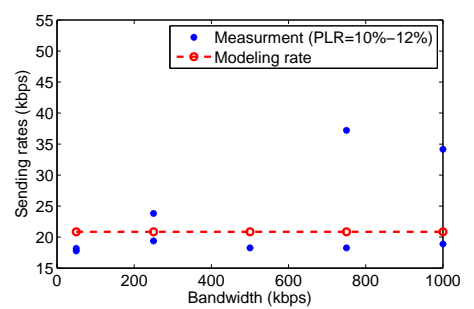

(b) Skype in CONS State

Fig. 4: Modeling sending rate with available bandwidth. PLR is from $0 \%$ to $10 \%$. The two-way propagation delay is fixed as $50 \mathrm{~ms}$.

where $C_{w}$ is the available bandwidth, $p$ is the packet loss rate, $\delta$ is the constant sending rate at CONS state, $\gamma$ and $\mu$ are two model parameters in NORM state.

To verify the model and derive the parameters, we measured Skype sending rates under a wide range of PLR and available bandwidth settings. Totally 25 scenarios are set up with the PLR varying from $0 \%-12 \%$ and the available bandwidth varying from $50 \mathrm{kbps}-1000 \mathrm{kbps}$. Figure 4 shows the measured data versus our proposed model. As illustrated in Figure 4(a), in NORM state, data points at each available bandwidth are overlapped or closely located to each other. A linear curve is sufficient to fit all the data points. In CONS state, most of the sending rates are around $21 \mathrm{kbps}$ as shown in Figure 4(b). With curve fitting over the measured data, the parameters are obtained as $\gamma=0.77, \mu=-10.8$ and $\delta=21$.

It is also interesting to observe how Skype's sending rate $R_{S}$ relates to its RTT. Figure 5 is the scatter plot of Skype RTT versus the surplus bandwidth $C_{w}-R_{S}$. Each point corresponds to one Skype run under some combination of PLR and available bandwidth. It can be seen that the RTT drops as the surplus bandwidth increases. We can model RTT as a function of the surplus bandwidth:

$$
R T T=\frac{\alpha}{C_{w}-R_{S}+\beta}+\sigma,
$$

where $\alpha, \beta$ and $\sigma$ are model parameters. The intuition behind this formula is that the system can be generally modeled as a $M / M / 1$ queue with some corrections on the traffic pattern. From the classic queueing theory, the per-packet queueing delay in a $M / M / 1$ queue with capacity $C$ and rate $R$ can be calculated as $\frac{B}{C-R}$, where $B$ is the average packet size. In other words, queueing delay is inversely proportional to the surplus bandwidth $C-R$ on a link. In (2), we can treat $\sigma$ as the static propagation delay component in RTT. $\beta$ controls the RTT increasing speed at low surplus bandwidth region when Skype tends to send a fairly large number of small packets. By curve fitting over the measured data, we find the parameters to be $\alpha=6906, \beta=64$, and $\sigma=54.63$, which match our system settings that propagation delay is $50 \mathrm{~ms}$, measured average packet size is 6364 bits. Therefore, with the sending rate model, it is possible to calculate RTT as a function of available bandwidth.

From the sending rate model, we summarize that Skype has two built-in rate control mechanisms: when PLR is less

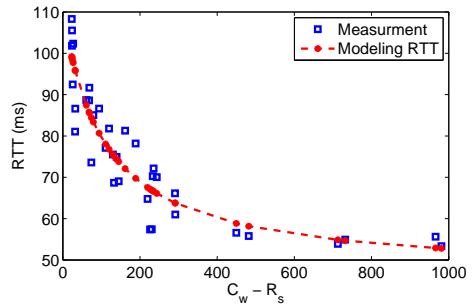

Fig. 5: Relation between Skype RTT and surplus bandwidth. Each point is for one combination of bandwidth and packet loss rate.

than $10 \%$, it controls its sending rate to maintain around $77 \%$ utilization of the available bandwidth; when PLR is greater than $10 \%$, it sends out data at a conservative constant rate.

\section{B. Video Rate}

Skype uses a video codec that is able to adapt its video bit rate to network conditions [9]. This is also verified in our measurements in Section IV-B. We notice that the video rate increases proportionally with the available bandwidth. Skype employs FEC coding to combat with packet losses. In Section IV-A, we observe that Skype decreases its video rate as PLR increases, but keeps its sending rate unchanged. This indicates that Skype uses more bandwidth to transmit FEC bits when PLR increases. Let $R_{v}$ be the actual video rate. We define the FEC redundancy ratio as

$$
\rho=\frac{R_{S}-R_{v}}{R_{S}} .
$$

When $\rho$ becomes larger, there are more redundancy bits.

To investigate the relation between FEC ratio and packet losses, we measured Skype's video rate and sending rate under various network conditions. FEC ratios are plotted as a function of PLR in Figure 6. Through simple linear regression, FEC ratio is modeled as a linear function of PLR:

$$
\rho=\psi+\omega p
$$

where $\psi$ and $\omega$ are constants determined by Skype's FEC mechanism. Through curve fitting, we found that $\psi=0.15$, $\omega=4.5$. The model curve is also illustrated in Figure 6. We observe that the model curve fits the measurement points very well. In (3), $\omega$ is the ratio between the FEC redundancy ratio and the packet loss rate. From the curve fitting results, we know that the redundancy ratio of Skpye is about 4.5 times of the actual loss rate. This is a very aggressive FEC protection.

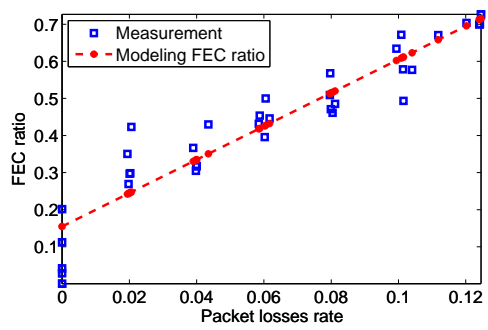

Fig. 6: FEC redundance ratio as a function of PLR. 
This result echos the conclusion in [7] that there is a significant space to improve Skype's FEC efficiency for voice calls.

Combining the sending rate model in (1) and the FEC model in (3), the video rate of Skype can be formulated as a function of the available bandwidth and PLR:

$$
R_{v}\left(C_{w}, p\right)= \begin{cases}(1-\psi-\omega p)\left(\gamma C_{w}+\mu\right) & p<10 \% \\ (1-\psi-\omega p) \delta & p \geq 10 \%\end{cases}
$$

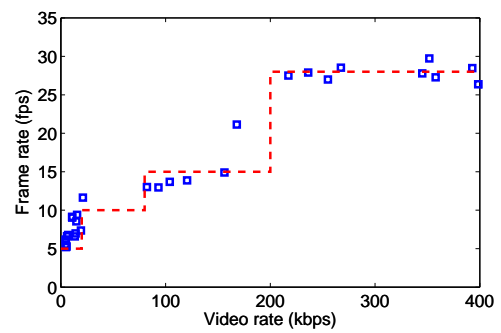

Fig. 7: Video frame rate as a function of video bit rate.

\section{Video Quality}

The ultimate QoE measure of video call service is the video quality perceived by users. We use a subjective quality model [18] to assess Skype's video quality. The videophone subjective quality model, also known as the opinion model for video-telephony applications, has been standardized as ITUT Recommendation G.1070 [19]. The perceived video quality is measured by the Mean Opinion Score (MOS), a subjective quality score that ranges from unacceptable to excellent. This model estimates the video quality affected by coding distortion and frame reduction. It is a function of video rate and frame rate. We rewrite the model as:

$$
Q=1+G\left(f, R_{v}\right),
$$

where $f$ is the frame rate measured in frames-per-second (fps), and $R_{v}$ is the video rate.

To assess the video quality of Skype, we need to measure Skype's frame rate under various network conditions. Since most video codec changes its frame rate based on the video rate, we investigate the relation between video rate and frame rate. We collect the frame rate and video rate information from the Skype window. The measured data are plotted in Figure 7. Again, each point corresponds to the measurement result under a combination of PLR and available bandwidth.

In Figure 7, we observe that most video frame rates are distributed around $5 \mathrm{fps}, 10 \mathrm{fps}, 15 \mathrm{fps}$, and $28 \mathrm{fps}$. As video rate increases, the frame rate increases. We conjecture that Skype codec encodes video into a pre-selected set of frame rates. It selects a frame rate according to the current video rate. Thus, we propose the frame rate model of Skype as a piecewise-constant function:

$$
f\left(C_{w}, p\right)=\left\{\begin{array}{ll}
5 & R_{v}\left(C_{w}, p\right) \leq 20 \\
10 & 20<R_{v}\left(C_{w}, p\right) \leq 80 \\
15 & 80<R_{v}\left(C_{w}, p\right) \leq 200 \\
28 & 200<R_{v}\left(C_{w}, p\right)
\end{array},\right.
$$

where the video rate is measured in kbps.

By combining the frame rate model in (6), the video rate model in (4) and the ITU-T MOS model in (5), the Skype's subjective quality can be calculated as:

$$
\begin{aligned}
& Q_{S}\left(C_{w}, p\right)=1+G\left(f\left(C_{w}, p\right), R_{v}\left(C_{w}, p\right)\right) \\
& =1+\left(c-\frac{c}{1+\left(R_{v}\left(C_{w}, p\right) / d\right)^{e}}\right) * \\
& \exp \left(-\frac{\left(\ln \left(f\left(C_{w}, p\right)\right)-\ln \left(a+b R_{v}\left(C_{w}, p\right)\right)\right)^{2}}{2\left(h+g R_{v}\left(C_{w}, p\right)\right)^{2}}\right),
\end{aligned}
$$

where the coefficients $a, b, c, d, e, h, g$ are model parameters as defined in ITU-T Recommendation G.1070 [19]: $a=1.431$, $b=0.02228, c=3.759, d=184.1, e=1.161, h=1.446$, $g=0.03881$.

\section{Model Validation}

To validate the above models, we conduct experiments where a Skype flow competes with background UDP traffic on a bottleneck link. The link's capacity is set to $700 \mathrm{kbps}$, and the propagation delay is fixed at $50 \mathrm{~ms}$. UDP traffic is injected into the test-bed at constant rate using the "iPerf" tool. After Skype enters into steady state, the sending rate, video rate, and frame rate are sampled. We carry out experiments under various scenarios with the UDP traffic rate varying from $0 k b p s$ to $600 \mathrm{kbps}$. Each experiment is run for 20 minutes and the sampling interval is one second. All measured points are illustrated as in Fig. 8.

We also use the proposed models to predict Skype's sending rate, video rate and frame rate. Given the link capacity is $C$ and the UDP rate is fixed at $R_{U}$, the available bandwidth for Skype can be expressed as:

$$
C_{w}=C-R_{U}
$$

Substituting (8) into the sending rate model $R_{S}\left(C_{w}, p\right)$ in (1), the video rate model $R_{v}\left(C_{w}, p\right)$ in (4), and the frame rate model $f\left(C_{w}, p\right)$ in (6), Skype's modeling rates are calculated. We compare them with the measurement results in Fig. 8. The figures show that our modeling curves match the measurement results pretty well. The Pearson correlation coefficients between the measurement and modeling results are 0.9898, 0.9831 , and 0.9545 for sending rate, video rate, and frame rate respectively.

\section{ANALYSis OF SKYPE PERFORMANCE UNDER COMPETITION}

Our measurement and modeling study characterize a Skype video call's behaviors in a controlled environment. In a real network, a Skype video call competes with other network flows, including other Skype calls and TCP flows, for network resources. In this section, we extrapolate from the obtained Skype rate control and quality models and quantitatively answer the following questions through numerical analysis:

1) How Skype video call users respond to quality degradation resulted from network impairments? How effective user back-offs are as a user-level rate control scheme? 


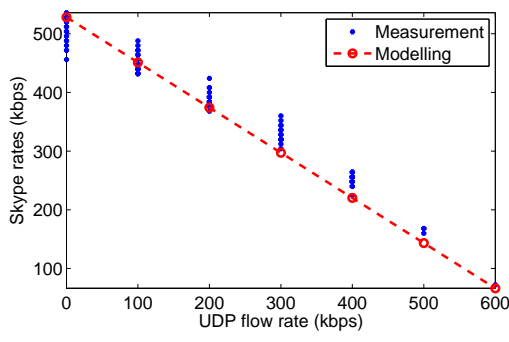

(a) Sending Rate

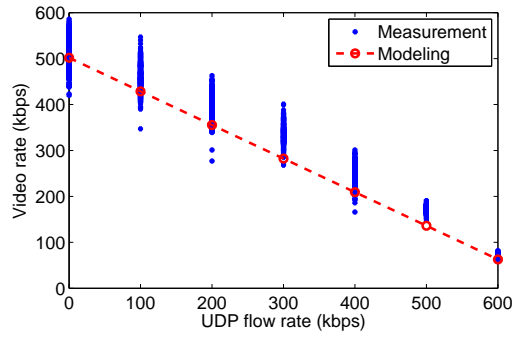

(b) Video Rate

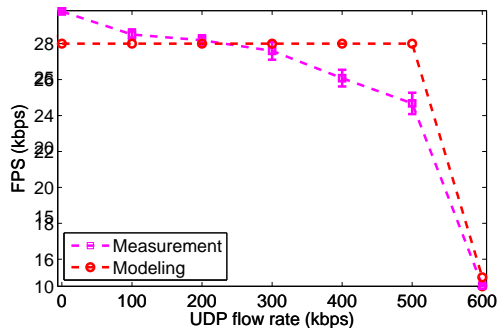

(c) Frame Rate

Fig. 8: Model validation. The capacity of bottleneck link is $700 \mathrm{kps}$, and the two-way propagation delay is fixed at $50 \mathrm{~ms}$.

2) What is the performance of a Skype video call when it competes with other Skype calls and TCP flows? Is Skype video call TCP-friendly?

\section{A. Network Model}

We consider a $4 G$ type of wireless cell where multiple TCP and Skype users compete for the access to the base-station. Both the uplink and downlink are the potential bandwidth bottlenecks. Due to the lossy nature of wireless transmission, we further assume that there are random channel losses on access links other than congestion losses. The base station takes traffic from a large number of users. It has been shown in [20] that when the link multiplexing degree is high, the packet arrival process can be well modeled as a Poisson process. We assume that an access link has finite buffer and model it as a $M / M / 1 / K$ queue, which is a drop-tail queue with a total buffer size of $K$. Let $C$ denote the total link capacity. Given the total traffic arrival rate of $R$, the average congestion loss probability $p_{q}$ and queueing delay $t_{q}$ can be approximately calculated as [21]:

$$
\begin{aligned}
p_{q} & =\frac{1-R / C}{1-(R / C)^{K+1}}\left(\frac{R}{C}\right)^{K} \\
t_{q} & =\frac{B(1-R / C)}{R\left(1-(R / C)^{K}\right)} \sum_{i=0}^{K} i\left(\frac{R}{C}\right)^{i},
\end{aligned}
$$

where $B$ is the average packet size. In our numerical analysis, we consider a LTE wireless network [22], with 100Mbps downlink capacity and 50Mbps uplink capacity. Since video call is bi-directional communication, all users within a cell compete for the 50Mbps uplink bandwidth.

TCP users react to congestion by adjusting their sending rates according to packet losses and round trip time. We assume all TCP users are long-lived flows whose congestion control phase is significant longer than the slow start phase. According to [23], for a TCP flow with end-to-end loss probability $p$ and delay $t$, the TCP sending rate can be characterized by:

$$
R_{T}=\frac{1.5 \sqrt{2 / 3} B_{T}}{t \sqrt{p}}
$$

where $t, q$ and $B_{T}$ represent round-trip delay, packet loss rate and TCP packet size respectively. The delay $t$ is the sum of queueing delay $t_{q}$ and propagation delay $t_{c}$. The end-to-end packet loss probability consists of two parts: $p=p_{q}+p_{c}$, where $p_{q}$ is the queueing loss due to congestion, and $p_{c}$ is the random channel loss. Our analysis can also be extended to incorporate short-lived TCP sessions following a different model [24]. We skip it here for the clarity of presentation.

\section{B. User-level Rate Control}

Previous studies [10], [11] have shown that user back-offs serve as an effective rate control scheme at the user-level. It can significantly reduce network traffic when the user QoE degrades as the network congestion builds up. In the current Skype implementation, it pops up a window to recommend users to turn off their video when the network conditions degrade. In this section, we use the obtained quality model in (7) to study the effectiveness of user back-offs as a userlevel rate control scheme for Skype video calls. Noted here, we consider a user dropping the video call if she turns off her video. The call session might still continue with just voice. Specifically, we assume that a user drops her video call probabilistically when the MOS score drops. The drop-off probability [19] is a function of the MOS score as enumerated in Table III.

TABLE III: Call drop ratio at different MOS scores

\begin{tabular}{|c|c|c|c|c|c|c|c|}
\hline MOS score & 1.5 & 2 & 2.5 & 3 & 3.5 & 4 & 4.5 \\
\hline Drop ratio & 0.98 & 0.85 & 0.7 & 0.5 & 0.3 & 0.1 & 0 \\
\hline
\end{tabular}

Given the available network bandwidth and packet loss rate, we can then calculate the video drop-off probability as follows:

$$
D_{S}\left(C_{w}, p\right)=D_{S}\left(Q_{S}\left(C_{w}, p\right)\right)
$$

To study the impact of video call drop-offs as a user-level rate control, let's assume there is a group of $N$ Skype users under a network condition characterized by $\left\{C_{w}, p\right\}$. The number of active users can be represented as $N\left(1-D_{S}\left(C_{w}, p\right)\right)$. The total effective traffic rate generated by all users can be approximated as:

$$
\hat{R}\left(C_{w}, p, N\right)=N\left(1-D_{S}\left(C_{w}, p\right)\right) R_{S}\left(C_{w}, p\right) .
$$

Considering the audio traffic is very small, and the average traffic contribution of each user is simply:

$$
\hat{R_{S}}\left(C_{w}, p\right)=\left(1-D_{S}\left(C_{w}, p\right)\right) R_{S}\left(C_{w}, p\right)
$$

We call $\hat{R_{S}}\left(C_{w}, p\right)$ the expected Skype sending rate. It represents the expected traffic generated by a Skype video call considering user drop-offs as a user-level rate control. For the 


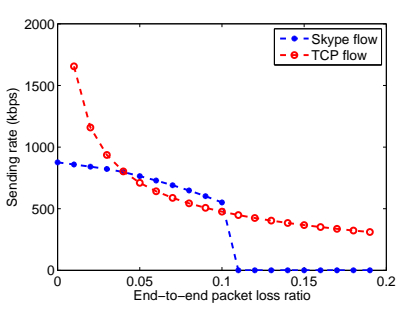

(a) Skype vs. TCP Rate

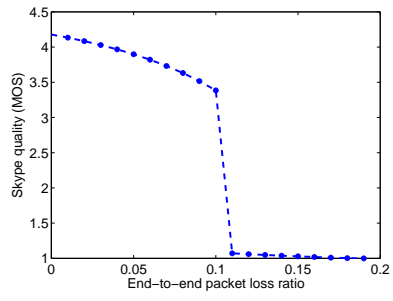

(b) Skype Quality

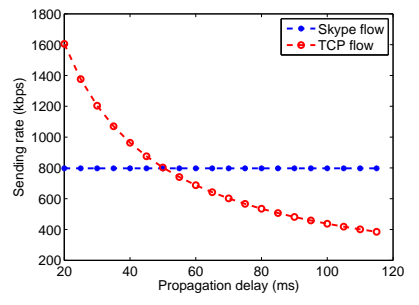

(c) Skype vs. TCP Rate

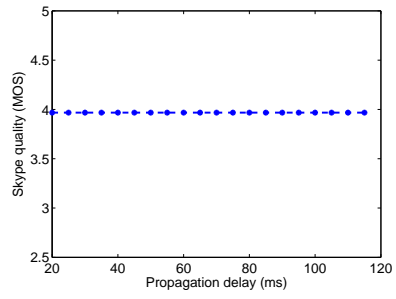

(d) Skype Quality

Fig. 9: Comparison of TCP and Skype's responsiveness to loss and delay: (a), (b) varying packet loss rate, the propagation delay is fixed at $60 \mathrm{~ms} ;$ (c), (d) varying propagation delay, the packet loss ratio is fixed at $4 \%$.

calculation in the rest of the paper, we will use $\hat{R}_{S}\left(C_{w}, p\right)$ in place of Skype's sending rate.

\section{TCP-friendliness of Skype Video Call}

Since Skype uses UDP as transport protocol, it has no congestion control scheme at the transport layer. To maintain the Internet stability, it is important for applications developed over UDP protocols to be TCP-friendly [23]. As demonstrated in the previous sections, Skype has its built-in rate control scheme at the application layer. Additionally, user back-offs can be considered as a rate control scheme at the user-level. We now study the TCP-friendliness of Skype video calls by taking into account rate control at both the application level and user level.

1) Responsiveness to Loss and Delay: We first compare a Skype video call's responsiveness to packet losses and delays with the responsiveness of a TCP flow. We not only compare the sending rates of Skype and TCP, but also compare the slope of their rate curves, which indicates how fast each flow reduces its rate under various network impairments. If the Skype curve has a steeper slope than the TCP curve, it means Skype is more responsive and reacts faster to network condition changes than TCP; if the Skype curve is flatter than TCP curve, it means Skype is more sluggish and reacts slower than TCP.

First, we introduce random packet losses at different PLR while keeping the end-to-end delay and available bandwidth fixed. The numerical results are illustrated in Figure 9.

In Figure 9(a), we plot the sending rate as end-to-end PLR increases from $0 \%$ to $20 \%$. The end-to-end delay is fixed at $60 \mathrm{~ms}$. TCP starts with a much higher rate than Skype. But TCP flow is more responsive to packet losses than Skype flow. When the end-to-end PLR increases from $1 \%$ to $3 \%$, the sending rate of TCP drops quickly. In contrast, when PLR is less than $10 \%$, Skype only slowly decreases its expected sending rate as defined in (13). As shown in Figure 9(b), Skype's video quality degrades as PLR increases. Skype's rate decreases in this region is due to user backoffs. The slope of the Skype curve is flatter than that of TCP. It shows that Skype is more sluggish in reducing its rate than TCP when the random PLR is below $10 \%$. When PLR goes over $10 \%$, Skype switches to the CONS state; both its sending rate and quality decrease significantly. In this region, Skype is much more conservative than TCP.

We also investigate how Skype and TCP adapt their rates to propagation delay. In Figure 9(c), we plot the sending rate of
Skype and TCP as we increase the two-way propagation delay from $20 \mathrm{~ms}$ to $120 \mathrm{~ms}$. Again, TCP has a much higher rate than Skype when the propagation delay is small. But TCP is more responsive to propagation delay increase than Skype. As propagation delay increases, TCP reduces its rate while Skype keeps its rate unchanged. We also notice in Figure 9(d), the video quality keeps stable. However, if we keep increasing the propagation delay till it become too large for realtime user interaction, most users will drop off, which will also cause Skype sending rate drop much faster than TCP.

2) Head-head Competition with TCP: In this section, we numerically study the behaviors of Skype video calls when they share a $4 \mathrm{G}$ wireless bottleneck link with TCP flows. The random channel loss rate is set at $p_{c}=2 \%$; the propagation delay is $t_{c}=50 \mathrm{~ms}$. There are totally $N_{T}$ TCP users and $N_{S}$ Skype users. Then the aggregate traffic rate is:

$$
R=N_{T} R_{T}(t, p)+N_{S}\left(1-D_{S}\left(C_{w}, p\right)\right) R_{S}\left(C_{w}, p\right),
$$

where $R_{T}$ is the sending rate calculated by the TCP rate model in (11). The available bandwidth seen by each Skype user can be determined by

$$
C_{w}=C-R+R_{S}\left(C_{w}, p\right)
$$

Equation (15) states that the available bandwidth seen by one Skype user equals the total bandwidth minus the aggregated TCP rates and Skype rates except the current one. By merging equations (1), (9), (10), (11), (12), (14), and (15), we reach at three equations for three unknowns $\left\{C_{w}, p_{q}, t_{q}\right\}$. We can numerically solve them for the rate allocation between TCP and Skype users and the video quality of Skype.

We calculate the bandwidth allocation among TCP flows and Skype flows as the number of flows in each group grows proportionally. We set the initial number of TCP and Skype users to one. Then we scale up their population by the same factor $k$. Figure 10 plots the bandwidth shares of a Skype and a TCP flow as $k$ increases.

In Figure 10(a), when the scale up factor $k \leq 20$, there is abundant bandwidth in the cell, and almost no congestion loss and delay. Skype operates in its NORM state and video quality is good. TCP only slightly drops its rate as $k$ increases. Congestion starts to build up as $k$ goes over 20, both queueing delay and packet loss increase as illustrated in Figure 10(c). TCP reacts faster to congestion and quickly reduces its sending rate. Meanwhile, as $k$ keeps increases, the available bandwidth 


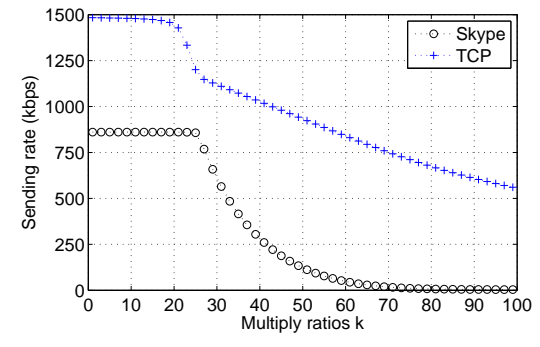

(a) Sending rate

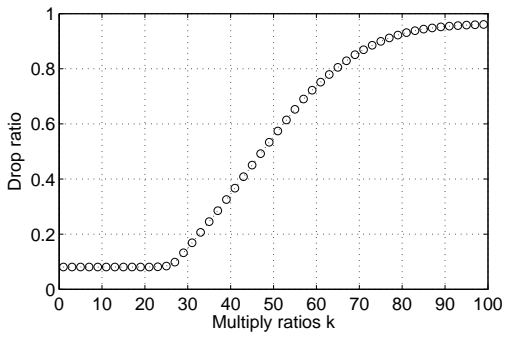

(b) Video drop-off ratio

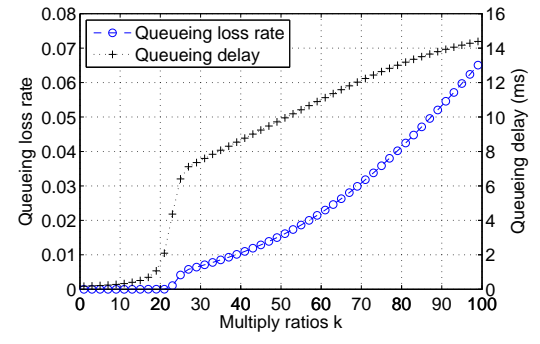

(c) Queue drops and delay

Fig. 10: Bandwidth allocation between Skype and TCP users as we scale up their population proportionally.

for each Skype call decreases, and the quality perceived by Skype users start to degrade. User video drop-offs kick in quickly as $k$ goes over 25, as shown in Figure 10(b). The expected sending rate of Skype decreases at a faster pace than TCP in Figure 10(a). TCP users take advantage of Skype user drop-offs and slow down their rate decreases when $k \geq 25$. Towards the end, all Skpy users turn off video and only the "persistent" TCP users prevail in the head-head bandwidth competition.

To summarize, when network congestion level is low, Skype is more sluggish than TCP and manages to provide smooth video transmission in face of mild random link losses and delays. When the network congestion level is high, Skype is more conservative and reacts faster to congestion than TCP, due to its built-in rate control scheme and user back-offs. This indicates that Skype is indeed TCP-Friendly when the network is heavily loaded and the congestion level is high.

\section{CONClusions AND Future WORK}

In this paper, we characterized the rate control schemes and video quality of Skype video calls. Through extensive measurement, we showed that Skype is robust against mild packet losses and propagation delays. It can efficiently utilize available network bandwidth when the network is lightly loaded. Skype significantly reduces its rate when the network impairments become severe. Based on measurement results, we developed rate control model, FEC model, and video quality model for Skype. Through numerical analysis, we showed that user back-offs serve as an effective user-level rate control scheme, and Skype is indeed "TCP-friendly". Our measurement results and models can also be used as quantitative tools in provisioning a network to support video calls with satisfactory user QoE.

As a future work, we will extend our study to include audio quality and its impact on user behaviors. We showed that Skype rate control is mostly driven by congestion delay and available bandwidth. We will explore more on delaydriven rate control algorithms for video telephony in wireline and wireless networks. We are also interested in improving the efficiency of Skype's FEC strategy. In this paper, we focused on the stationary behaviors of Skype. The transient behaviors of Skype under competition with other flows is also an interesting topic for future study. Finally, the rate control and video quality of Skype's multi-party video conferencing is also on our future research agenda.

\section{REFERENCES}

[1] Skype Inc., "Skype features," http://www.skype.com.

[2] S. A. Baset and H. G. Schulzrinne, "An analysis of the skype peer-topeer internet telephony protocol," in Proceedings of IEEE INFOCOM, April 2006, pp. 1-11.

[3] D. Bonfiglio, M. Mellia, M. Meo, N. Ritacca, and D. Rossi, "Tracking down skype traffic," in Proceedings of IEEE INFOCOM, Apr 2008, pp. 261-265.

[4] S. Guha, N. Daswani, and R. Jain, "An Experimental Study of the Skype Peer-to-Peer VoIP System," in Proceedings of the 5th International Workshop on Peer-to-Peer Systems, Santa Barbara, CA, February 2006, pp. 1-6.

[5] K. Chen, T. Huang, P. Huang, and C. Lei, "Quantifying skype user satisfaction," in Proceedings of ACM SIGCOMM, vol. 36, Oct 2006.

[6] T. Huang, K. Chen, and P. Huang, "Tuning skype redundancy control algorithm for user satisfaction," in Proceedings of IEEE INFOCOM, April 2009, pp. 1179-1185.

[7] T. yuan Huang, K. ta Chen, and P. Huang, "Could skype be more satisfying? a QoE-Centric study of the fec mechanism in an internetscale voip system," IEEE Network, vol. 24, no. 2, p. 42, Mar 2010.

[8] L. D. Cicco, S. Mascolo, and V. Palmisano, "A mathematical model of the skype voip congestion control algorithm," in Proceedings of IEEE Conference on Decision and Control, Dec 2008.

[9] - "Skype video congestion control: an experimental investigation," Computer Networks, vol. 55, no. 3, pp. 558 - 571, Feb 2011.

[10] T. Bu, Y. Liu, and D. Towsley, "On the tcp-friendliness of voip traffic," in Proceedings of IEEE INFOCOM, Dec 2006.

[11] R. Morris and Y. Tay, "A model for analyziing the roles of network and user behavior in congestion control," Massschusetts Institute of Technology, Tech. Rep., May 2004.

[12] Microsoft Resarch Asia, "Network Emulator for Windows Toolkit (NEWT)," http://blogs.msdn.com/b/lkruger.

[13] e2eSoft, "Vcam: Webcam emulator," http://www.e2esoft.cn/vcam/.

[14] Renovation Software, "Text grab for windows," http://www. renovation-software.com/en/text-grab-sdk/textgrab-sdk.html.

[15] Google Inc., "On2 video codec," http://www.on2.com/on2-video/.

[16] M. Jain and C. Dovrolis, "Pathload: A measurement tool for endto-end available bandwidth," in Proceedings of Passive and Active Measurements (PAM) Workshop, 2002, pp. 14-25.

[17] N. Hu, L. E. Li, Z. M. Mao, P. Steenkiste, and J. Wang, "Locating internet bottlenecks: Algorithms, measurements, and implications," 2004

[18] K. Yamagishi and T. Hayashi, "Qrp08-1: Opinion model for estimating video quality of videophone services," in Proceeding of IEEE Global Telecommunications Conference, Dec 2006, pp. 1-5.

[19] ITU-M, "G.1070: Opinion model for video-telephony applications," ITU, Tech. Rep., Apr 2007.

[20] J. Cao and K. Ramanan, "A possion limit for buffer overflow probabilities," in Proceedings of IEEE INFOCOM, Mar 2002.

[21] L. Kleinrock, Theory, Volume 1, Queueing Systems. Wiley-Interscience, 1975.

[22] ITU-R, "Recommendation m.1645: Framework and overall objectives of the future development of imt-2000 and systems beyond imt-2000," ITU, Tech. Rep., Jun 2003.

[23] S. Floyd and K. Fall, "Promoting the use of end-to-end congestion control in the internet," Networking, IEEE/ACM Transactions on, vol. 7, no. 4, pp. 458 -472, Aug 1999.

[24] N. Cardwell, S. Savage, and T. Anderson, "Modelling the performance of short tcp connections," University of Washington, Tech. Rep., Nov 1998. 